\title{
Confidence Limit for the Existence of a Solution to the One-Dimensional Magnetotelluric Inverse Problem
}

\author{
Yuanchou $\mathrm{ZHANG}^{1 *}$ and Ken PAULSON ${ }^{2}$ \\ ${ }^{1}$ Geophysics Division, Department of Physics, University of Toronto, Ontario, Canada \\ ${ }^{2}$ Cybernetics Laboratory, University of Saskatchewan, Saskatoon, Canada
}

(Received January 24, 1996; Revised May 8, 1997; Accepted June 7, 1997)

\begin{abstract}
The necessary and sufficient conditions for the existence of a solution to the one-dimensional magnetotelluric inverse problem are extended to error data. The confidence criterion is defined and used for testing the existence of a one-dimensional solution when data errors are considered. Synthetic data sets and two very popular real data sets are tested using this new confidence criterion.
\end{abstract}

\section{Introduction}

Weidelt (1972, 1986), Parker (1980), Yee and Paulson (1988a) stipulated the necessary and sufficient conditions for the existence of a solution to the one-dimensional (1D) magnetotelluric inverse problem. The existence was based on an all-inclusive set of continuous and discontinuous one-dimensional conductivity profiles, called $\bar{S}^{+}$(Yee and Paulson, 1988b), and is guaranteed if two simple constraints on the c-response data set $\left\{c\left(\nu_{j}\right)\right\}_{1}^{N}$ are satisfied. Here, $c\left(\nu_{j}\right)$ is the cresponse at the real, positive, discrete frequency $\nu_{j}$. From this data set, a pair of $N \times N$ Hermitian test matrices, $\mathbf{Q}$ and $\overline{\mathbf{Q}}$, are constructed, with typical elements

$$
\left[q_{j k}\right]=\left[\frac{i\left(c\left(\nu_{j}\right)-c^{*}\left(\nu_{k}\right)\right)}{\nu_{j}+\nu_{k}}\right],
$$

and

$$
\left[\bar{q}_{j k}\right]=\left[\frac{\nu_{j} c\left(\nu_{j}\right)+\nu_{k} c^{*}\left(\nu_{k}\right)}{\nu_{j}+\nu_{k}}\right], \text { for } j, k=1,2, \cdots, N,
$$

where $*$ denotes complex conjugation. If both of these test matrices are positive definite, the given data set is consistent with some $c(\nu), \nu_{1} \leq \nu \leq \nu_{N}$, corresponding to a one-dimensional conductivity, $\sigma \in \bar{S}^{+}$. If only certain sub matrices are found to be positive definite, consistency with some $c(\nu)$ would be restricted to the frequency ranges involved in the definition of those sub matrices.

The derivation of Weidelt $(1972,1986)$, Yee and Paulson (1988a) is applicable only to precise data and consequently is of little practical use. While Parker (1980), Weidelt (1990) considered data imprecision, the former was only through use of a chi-square goodness of fit to a 1D conductivity distribution in $D^{+}$(i.e., a sequence of positive delta functions) which requires that the residuals have a Gaussian distribution, and the latter failed on the generalization for $N$ frequencies.

In this paper it will be shown that compensation for the effects of random noise is possible allowing one to test for the one-dimensionality of the conductivity structure associated with any measured response data set $\left\{c\left(\nu_{j}\right)\right\}_{1}^{N}$ that contains random noise in its structure. To this end, it will be assumed that the errors in both the real and imaginary parts of each $c\left(\nu_{j}\right)$ is known. These

*Formerly with Cybernetics Laboratory, University of Saskatchewan, Saskatoon, Canada. 
errors can be either in the form of standard deviations or maximum errors and should always be part of any experimental magnetotelluric data set. This allows the definition of two error matrices $\Delta$ and $\bar{\Delta}$ associated with $\mathbf{Q}$ and $\bar{Q}$, respectively. Then a pair of Hermitian perturbation matrices, $\mathbf{P}$ and $\overline{\mathbf{P}}$ are defined which are just sufficient to force the sums $\mathbf{Q}+\mathbf{P}$ and $\overline{\mathbf{Q}}+\overline{\mathbf{P}}$ to become positive definite. Finally, a criterion is chosen which relates these minimum perturbation matrices to their corresponding error matrices. Obviously, if the perturbation elements are large in comparison to the error elements, there are factors other than imprecision causing the non-positive-definiteness of the test matrix. To this end, non-negative scalars, $\mu$ and $\bar{\mu}$ are introduced, together with the ad hoc inequalities, $\mu \leq 1$ and $\bar{\mu} \leq 1$ as criteria for concluding that the measured response data set is compatible with some $1 \mathrm{D}$ conductivity structure.

The method has been thoroughly tested on both synthetic and measured data sets some of which are reproduced in the paper.

\section{Theoretical Considerations}

By the spectral theorem (Strang, 1980), every Hermitian matrix, $\mathbf{Q}$ may be diagonalized by some unitary matrix $\mathbf{U}$, such that

$$
\mathbf{U}^{H} \mathbf{Q} \mathbf{U}=\mathbf{U}^{-1} \mathbf{Q} \mathbf{U}=\mathbf{\Lambda}
$$

and may be decomposed into the sum

$$
\mathbf{Q}=\mathbf{U} \mathbf{\Lambda} \mathbf{U}^{H}=\sum \lambda_{j} \mathbf{u}_{j}^{H} \mathbf{u}_{j}
$$

where the elements $\lambda_{j}$ are the real eigenvalues of $\mathbf{Q}$, the column vectors $\mathbf{u}_{j}$ are the corresponding eigenvectors, and $H$ denotes the operation of complex-conjugate transpose.

In the spectral decomposition of $\mathbf{Q}$, the unitary matrix $\mathbf{U}$ may be chosen so that the $N$ elements of the diagonal matrix, $\boldsymbol{\Lambda}$, are in decreasing order. Thus,

$$
\lambda_{1} \geq \lambda_{2} \geq \ldots \geq \lambda_{N}
$$

If all of these eigenvalues are positive, $\mathbf{Q}$ is positive definite and the one-dimensional requirement of the test matrix in Eq. (1) is satisfied.

However, suppose that some of the eigenvalues are negative, where rank $N$ is assumed. Then, with the same ordering as before,

$$
\lambda_{h}>0, \lambda_{j}<0, \text { for } h=1, \cdots, p ; j=p+1, \cdots, N .
$$

This matrix, of course, is not positive definite.

Now consider a real diagonal matrix, $\mathbf{B}$, with real, non-negative, diagonal elements $(0,0, \cdots$, $\left.0, b_{p+1}, \cdots, b_{N}\right)$, where the $\left\{b_{j}\right\}$ elements are chosen so that

$$
b_{j}+\lambda_{j}>0, \text { for } j=p+1, \cdots, N
$$

Then,

$$
\mathbf{U}(\boldsymbol{\lambda}+\mathbf{B}) \mathbf{U}^{H}=\mathbf{U} \mathbf{\Lambda} \mathbf{U}^{H}+\mathbf{U B} \mathbf{U}^{H}=\mathbf{Q}+\mathbf{P},
$$

where the matrix $\mathbf{P}$ is chosen to be the minimum perturbation needed to transform $\mathbf{Q}$ into the positive definite matrix $\mathbf{Q}+\mathbf{P}$.

The perturbation, $\mathbf{P}$, may result because of random noise or the departure of the conductivity structure from one dimensionality. It may also arise because of other factors such as source effects 
and near-surface conductivity anomalies. In order to weight the contribution of random noise to the perturbation matrix, evaluate the scalar ratio

$$
\mu=\max \left[\frac{\left|\Re\left(p_{j k}\right)\right|}{\Re\left(\Delta_{j k}\right)}, \frac{\left|\Im\left(p_{j k}\right)\right|}{\Im\left(\Delta_{j k}\right)}\right], \forall j, k,
$$

where $\Re\{$.$\} and \Im\{$.$\} are the real and imaginary parts, respectively, of matrix elements from the$ perturbation matrix, $\mathbf{P}$, and the error matrix, $\Delta$. The error matrix is derived from the error in $c_{j}, j=1, \ldots, N$, and defined as:

$$
\Delta=\left[\frac{\left(\Im\left(\Delta c_{j}\right)+\Im\left(\Delta c_{k}\right)\right)+i\left(\Re\left(\Delta c_{j}\right)+\Re\left(\Delta c_{k}\right)\right)}{\omega_{j}+\omega_{k}}\right]
$$

In a similar manner, associated with the test matrix $\overline{\mathbf{Q}}$ (Eq. 2), there is an error matrix, $\bar{\Delta}$, a perturbation matrix $\overline{\mathbf{P}}$, and a scalar ratio $\bar{\mu}$. If both $\mu \leq 1$ and $\bar{\mu} \leq 1$, it will be concluded that the perturbation matrices needed to change $\mathbf{Q}$ and $\overline{\mathbf{Q}}$ into positive definite forms are within the limits of the error matrices $\Delta$ and $\bar{\Delta}$ and that the underlying conductivity structure is onedimensional. If these two conditions are not satisfied, the structure may not be one-dimensional. The word "may" is used because other factors besides noise and higher-order dimensionality can cause the tests to fail.

\section{Application to Synthetic Data}

\subsection{Synthetic example I:}

Consider a single plane layer of thickness $10 \mathrm{~km}$ and resistivity $100 \Omega \mathrm{m}$, over a half space of resistivity $10 \Omega \mathrm{m}$. The values of the c-response at different frequencies are readily calculable. Four of these values are shown in Table 1 and they have been used to construct the $4 \times 4$ matrices $\mathbf{Q}$ and $\overline{\mathbf{Q}}$ defined by Eqs. (1) and (2). Since the eigenvalues,

$$
\text { eigenvalue }(\mathbf{Q})=\left(40450 ., 158.93,2.7040 \times 10^{-1}, 2.7601 \times 10^{-4}\right)
$$

and

$$
\text { eigenvalue }(\overline{\mathbf{Q}})=\left(38516 ., 65.056,1.4038 \times 10^{-1}, 1.6018 \times 10^{-4}\right)
$$

are real and positive, both matrices are positive definite as they should be for a one-dimensional structure. However if the same c-response data are truncated to three significant figures, the eigenvalues become

$$
\text { eigenvalue }(\mathbf{Q})=\left(40473 ., 147.02,2.0692 \times 10^{-1},-3.0094 \times 10^{-1}\right),
$$

and

$$
\text { eigenvalue }(\overline{\mathbf{Q}})=\left(38607 ., 72.401,6.7893 \times 10^{-2},-2.3941 \times 10^{-1}\right) \text {. }
$$

Table 1. Synthetic c-response values for a $1 \mathrm{D}$ structure.

\begin{tabular}{|c|c|r|c|}
\hline Index $(j)$ & $\nu_{j}(\mathrm{mHz})$ & $\Re\left[c_{j}\right](\mathrm{m})$ & $\Im\left[c_{j}\right](\mathrm{m})$ \\
\hline 1 & 65.21 & 10254.765593 & -5146.990877 \\
\hline 2 & 75.00 & 9854.153611 & -5079.056625 \\
\hline 3 & 86.25 & 9445.785107 & -5026.055535 \\
\hline 4 & 99.18 & 9027.146524 & -4983.909007 \\
\hline
\end{tabular}


Since both $\mathbf{Q}$ and $\overline{\mathbf{Q}}$ have negative eigenvalues, the test matrices fail to satisfy the requirement for a one-dimensional structure.

The two error matrices in this case are readily determined from the discarded remainders of the truncated c-response values. Thus,

$$
\Delta=\left[\begin{array}{cccc}
9.761+14.64 i & 9.080+17.02 i & 9.456+12.60 i & 10.64+8.712 i \\
9.080+17.02 i & 8.488+19.09 i & 8.883+14.80 i & 10.05+10.96 i \\
9.456+12.60 i & 8.883+14.80 i & 9.226+11.07 i & 10.29+7.724 i \\
10.64+8.712 i & 10.05+10.96 i & 10.29+7.724 i & 11.23+4.813 i
\end{array}\right]
$$

and

$$
\bar{\Delta}=\left[\begin{array}{cccc}
4.000+6.000 i & 4.000+7.604 i & 4.569+5.999 i & 5.809+4.190 i \\
4.000+7.604 i & 4.000+9.000 i & 4.534+7.395 i & 5.708+5.583 i \\
4.569+5.999 i & 4.534+7.395 i & 5.000+6.000 i & 6.069+4.395 i \\
5.809+4.190 i & 5.708+5.583 i & 6.069+4.395 i & 7.000+3.000 i
\end{array}\right]
$$

The minimum perturbation matrices needed to create positive definite test matrices are

$$
\mathbf{P}=10^{-3} \times\left[\begin{array}{cccc}
58.10 & -104.1+9.791 i & 33.88-35.14 i & 11.42+25.46 i \\
-104.1-9.791 i & 188.4 & -66.67+57.30 i & -16.19-47.58 i \\
33.88+35.14 i & -66.67-57.30 i & 41.01 & -8.741+21.76 i \\
11.42-25.46 i & -16.19+47.58 i & -8.741-21.76 i & 13.41
\end{array}\right]
$$

and

$$
\overline{\mathbf{P}}=10^{-3} \times\left[\begin{array}{cccc}
51.56 & -82.20-25.09 i & -3.834+33.15 i & 34.01-7.260 i \\
-82.05+25.09 i & 142.7 & -10.03-54.63 i & -50.59+28.10 i \\
-3.834-33.15 i & -10.03+54.63 i & 21.60 & -7.197-21.33 i \\
34.01+7.260 i & -50.59-28.10 i & -7.197+21.33 i & 23.46
\end{array}\right]
$$

From the error and perturbation matrices the two scalar ratios may be evaluated from Eq. (6) giving

$$
\mu=0.0222
$$

and

$$
\bar{\mu}=0.0357 \text {. }
$$

Since both ratios are less than unity, it may be concluded that within the limits of the random noise the c-response data set resulted from some one-dimensional resistivity structure, which, of course, is the correct conclusion.

\subsection{Synthetic example II:}

Following Yee et al. (1988), a single horizontal component of a noise-free, time-dependent, magnetic-field signal was generated by an autoregressive-moving average and the corresponding orthogonal, noise-free, electric-field signal was generated based on the previously employed model of a single plane layer over a half-space. Two independent realizations of random noise with identical standard deviations were then added to each of these signals to create magnetic- and 
electric-field message sequences each containing 16,384 elements with 1-s sampling intervals. Each of these synthetic data components were then divided into 16 disjoint intervals each containing 1024 elements. Discrete Fourier transform sequences, $\left\{\tilde{H}^{(m)}(\nu)\right\}$ and $\left\{\tilde{E}^{(m)}(\nu)\right\}$ were calculated for the magnetic and electric fields within each of the 16 disjoint intervals. Point estimates of the impedance sequences, $\left\{\tilde{\tilde{Z}}^{(m)}(\nu)\right\}$ were then evaluated according to the relation

$$
\hat{\tilde{Z}}^{(m)}(\nu)=\frac{\tilde{E}^{(m)}(\nu)}{\tilde{H}^{(m)}(\nu)}, \quad m=1,2, \cdots, 16 ; \nu=0,1, \cdots, 1023 .
$$

These estimates are realizations of random variables because they are quotients of physical realizations of fields which have random elements in their structures. Because the standard deviations of the random-noise realizations are known, the standard deviations of each impedance estimate may be determined.

Since the model is one-dimensional, the impedance estimates and their standard deviations may be converted into c-response estimates. The sixteen c-response estimates at a frequency of $75 \mathrm{mHz}$ are plotted in Fig. 1 as small solid circles. The two largest circles in the figure are centered on two of these estimates and possess radii equal to the standard deviations of each estimate. Note that the circles have slightly different radii. This is so because the deterministic signal components of the fields vary over the 16 disjoint time intervals.

The arithmetic (sample) mean of the 16 impedance estimates, together with its standard deviation may be computed to yield an improved estimate of the impedance. These two values, converted to c-responses, are displayed in Fig. 1 as a triangle and its associated standard deviation circle. Theoretically, as the sample size is increased from 16 to infinity, the estimate provided by the arithmetic mean approaches the true value. The true value of the c-response is shown in Fig. 1 as a large solid circle. Note that it falls outside the circle of one standard deviation radius

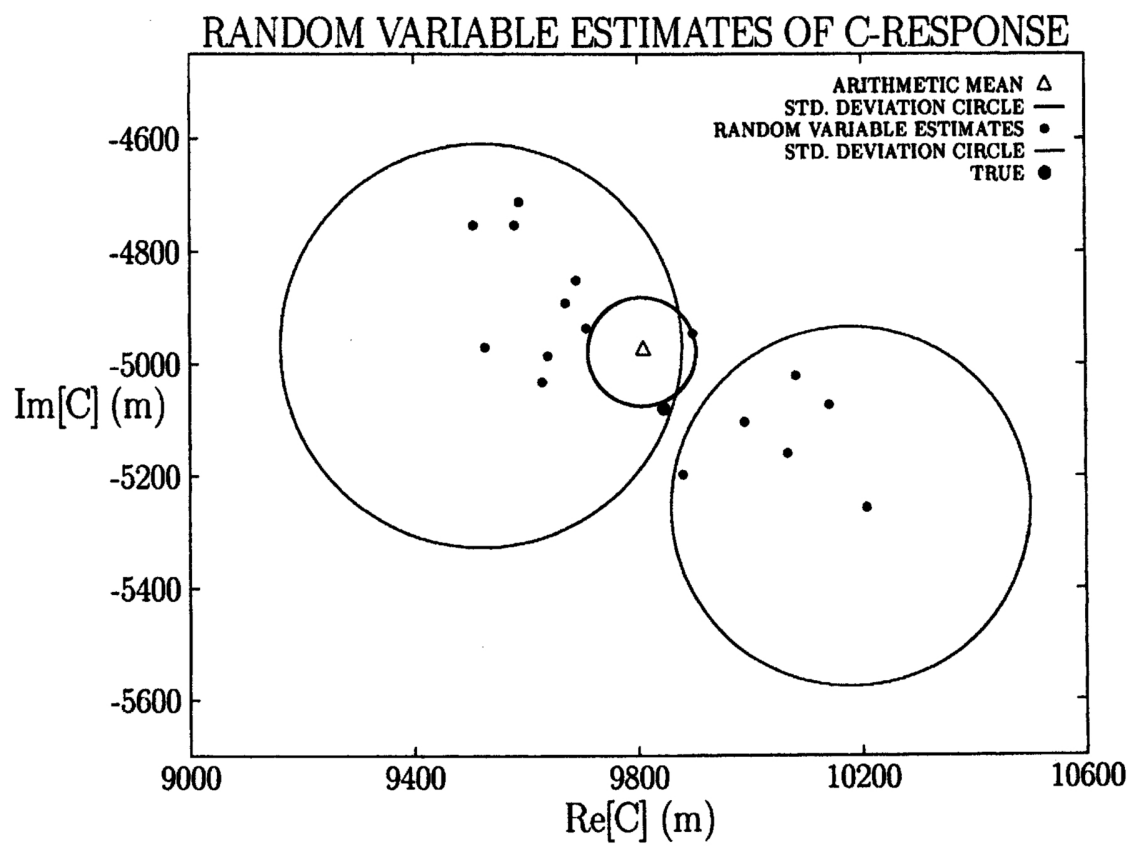

Fig. 1. The 16 c-response estimates and their associated standard deviations at frequency $75 \mathrm{~Hz}$. 
about the arithmetic mean estimate.

The arithmetic mean estimates together with their standard deviation circles for the four frequencies in Table 1 were used to prepare the test matrices $\mathbf{Q}$ and $\overline{\mathbf{Q}}$ and the error matrices $\Delta$ and $\bar{\Delta}$. Note that the entries in the error matrices are now standard deviation values and not maximum errors as used previously. The eigenvalues of $\mathbf{Q}$ and $\mathbf{Q}$,

$$
\text { eigenvalue }(\mathbf{Q})=(40853 ., 100.42,-0.03588,-65.905),
$$

and

$$
\text { eigenvalue }(\overline{\mathbf{Q}})=(38637 ., 118.95,0.01605,-12.364),
$$

each have negative entries and, therefore, the test of Yee and Paulson (1988a) would result in the conclusion that the underlying resistivity structure is not one dimensional.

However, computation of the minimum perturbation matrix allows evaluations of

$$
\mu=0.1287
$$

and

$$
\bar{\mu}=0.0567 \text {. }
$$

Once again, to within the limits of the inherent noise, it is concluded that the resistivity structure is one dimensional.

\section{Application to Experimental Data}

The theory developed in this paper will now be applied to magnetotelluric data sets obtained by Larsen (1975) and by Jones and Hutton (1979). Both data sets consist of a single pair of apparent resistivity and phase sequences, together with estimates of their standard deviations. The Larsen data set was converted to c-response values by Parker and Whaler (1981). We, in turn, have converted the Jones and Hutton data to c-response values using

$$
c(\nu)=\frac{Z(\nu)}{i 2 \pi \nu \mu_{0}}=\frac{\sqrt{\rho_{a}(\nu)} e^{i \phi_{a}(\nu)}}{i \sqrt{2 \pi \nu \mu_{0}}},
$$

where $\rho_{a}(\nu)$ and $\phi_{a}(\nu)$ are, respectively, the apparent resistivity and phase measured at frequency $\nu, \mu_{0}$ is the magnetic permeability of free space and $i=\sqrt{-1}$. The error associated with $c(\nu)$ may be calculated in the following way:

$$
\Delta c(\nu)=\left(\frac{\Delta \rho_{a}(\nu)}{2 \rho_{a}(\nu)}+i \Delta \phi_{a}(\nu)\right)|c(\nu)| e^{\phi_{c}(\nu)},
$$

where $\phi_{c}(\nu)$ represents the phase of $c(\nu)$. There is $\pi / 2$ difference between $\phi_{c}(\nu)$ and $\phi_{a}(\nu)$ or $\phi_{c}=\phi_{a}-\pi / 2$. The Eq. (21) represents an error ellipse and the two radii are equal to $|c(\nu)| \Delta \rho_{a}(\nu) /\left(2 \rho_{a}(\nu)\right)$ and $|c(\nu)| \Delta \phi_{a}(\nu)$ respectively with one axis lies on $\phi_{c}(\nu)$.

Strictly speaking, of course, the conversion in Eq. (20) assume that the underlying structure is one dimensional, but one can do little else in these cases where the full impedance tensor was not measured in the first place. If the structure, indeed, is not one dimensional, the conversion will only worsen the departure from a one-dimensional structure as reflected in our test matrices. In any case, the problem could be cleared up entirely by making a small change in the definition of the c-response function, namely,

$$
\tilde{c}(0, \nu)=\left(\frac{-\tilde{E}_{i}}{(\nabla \times \tilde{\mathbf{E}})_{j}}\right)_{z=0},
$$


where $i, j$ is some combination of $x, y$ and the other symbols on the right-hand side are conventional ones used in magnetotelluric theory.

Both Larsen (1975) and Parker and Whaler (1981) concluded that the seven lowest frequencies of the Larsen data set could be adequately interpreted by a 1D conductivity model. However, Yee and Paulson (1988a) concluded that only certain combinations of 3-tuples of frequency values were interpretable as being consistent with some 1D distribution and that there were no combinations of 4 -tuples that would satisfy this criterion. We have calculated the scalar indices for the seven lowest frequencies and find that

$$
\mu=0.09664
$$

and

$$
\bar{\mu}=0.06446 .
$$

Since the two indices are much smaller than unity, we conclude that the seven lowest frequency values lowest frequency responses are consistent with some $1 \mathrm{D}$ conductivity distribution to within the limits of the inherent random noise. Extending the analysis to include the lowest 8,9 and 12 frequency values gives values for the scalar indices given in Table 2. From this analysis, we conclude that $1 \mathrm{D}$ consistency may be extended to the 8 th lowest frequency value, but not beyond.

Table 2. Scalar ratios for Larsen's data set for different numbers of data points (starting from the lowest frequency).

\begin{tabular}{|c|c|c|c|c|}
\hline Number of data points & 7 & 8 & 9 & 12 \\
\hline$\mu$ & 0.09664 & 0.5245 & 1.877 & 15.43 \\
\hline $\bar{\mu}$ & 0.06446 & 0.06624 & 0.1742 & 13.38 \\
\hline
\end{tabular}

For the Jones and Hutton data set, different combinations of c responses at different frequencies have been analysed. The largest consistent frequency region occurred for the highest frequencies, between 4 and $35 \mathrm{mHz}$, a span of 8 frequency values, for which $\mu=0.2404$ and $\bar{\mu}=0.02858$. When the next lowest frequency was added, the scalar indices for $N=9$ became $\mu=3.217$ and $\bar{\mu}=0.06182$. The conclusion is that the data set is consistent with some $1 \mathrm{D}$ distribution over a frequency range of 3.13 octaves $(4$ to $35 \mathrm{mHz}$ ) and that the data associated with the lower frequencies of the data set are not.

\section{Conclusions}

The testing for the one dimensionality of a given magnetotelluric c-response data set may be heavily influenced by the imprecision in the data set. We have presented a theory whereby the noise inherent in the data, as expressed by either the maximum error or the standard deviation, may be used to compensate for the imprecision. The theory is an extension to the deterministic analysis of Weidelt (1986), Yee and Paulson (1988a).

The theory has been applied to both synthetic and experimental data sets with satisfactory results.

Since the scalar indices adopted to determine if a data set is compatible with some $1 \mathrm{D}$ conductivity distribution to within limitations of the noise is purely an ad hoc one, the entire subject should be re-examined. However, the approach described here is a substantial improvement over what has been done in the past. 
We would like to thank Drs. Yoshi Honkura, Hiroaki Toh and Peter Weidelt for their valuable comments and suggestions which improved the present manuscript. Helpful communications with Dr. H. Yin (Dept. of Mathematics and Statistics, Univ. of Saskatchewan) are acknowledged with gratitude. Drs. W. Zawalski and T. Zhu carefully read the original and revised versions of the manuscript, and made it readable on many points. The second revision was done after the first author (Y.Z.) jointed Geophysics Division, Dept. of Physics, University of Toronto. Drs. G. F. West and R. C. Bailey's kind help is greatly appreciated.

\section{REFERENCES}

Jones, A. G. and R. Hutton, A multi-station magnetotelluric study in southern Scotland-II. Monte-Carlo inversion of the data and its geophysical and tectonic implications, Geophys. J. R. astr. Soc., 56, 351-368, 1979.

Larsen, J. C., Low frequency $(0.1-6.0 \mathrm{cpd})$ electromagnetic study of deep mantle electrical conductivity beneath the Hawaiian Islands, Geophys. J. R. astr. Soc., 43, 17-46, 1975.

Parker, R. L., The inverse problem of electromagnetic induction: existence and construction of solutions based upon incomplete data, J. Geophys. Res., 85, 4421-4425, 1980.

Parker, R. L. and K. A. Whaler, Numerical methods for establishing solutions to the inverse problem of electromagnetic induction, J. Geophys. Res., 86, 9574-9584, 1981.

Strang, G., Linear Algebra and its Applications, 414 pp., Academic Press, New York, 1980.

Weidelt, P., The inverse problem of geomagnetic induction, Z. Geophys., 38, 257-289, 1972.

Weidelt, P., Discrete frequency inequalities for magnetotelluric impedances of one-dimensional conductors, $J$. Geophys., 59, 171-176, 1986.

Weidelt, P., 1D-Konsistenzbereiche für Übertragungsfunktionen mit Fehlern, in Protokoll über das 13. Kolloquium "Elektromagnetische Tiefenforschung", Hannover, 1990.

Yee, E. and K. V. Paulson, Necessary and sufficient conditions for the existence of a solution to the one-dimensional magnetotelluric inverse problem, Geophys. J. R. astr. Soc., 93, 279-293, 1988a.

Yee, E. and K. V. Paulson, Properties of the c-response function for conductivity distributions of class $\bar{S}^{+}$, Geophys. J. R. astr. Soc., 93, 265-278, 1988 b.

Yee, E., P. R. Kosteniuk, and K. V. Paulson, The reconstruction of the magnetotelluric impedance tensor: an adaptive parametric time-domain approach, Geophysics, 53, 1080-1087, 1988. 